Article

\title{
Towards a New, Pre-Clinical, Subject-Independent Test Model for Kinematic Analysis after Total Knee Arthroplasty-Influence of the Proximo-Distal Patella Position and Patellar Tendon Stiffness
}

\author{
Adrian Sauer ${ }^{1,2, *} \mathbb{D}$, Allan Maas ${ }^{1,2}\left(\mathbb{D}\right.$, Svenja Ottawa ${ }^{1,3}$, Alexander Giurea ${ }^{4}$ and Thomas M. Grupp ${ }^{1,2}$ \\ 1 Research and Development, Aesculap AG, 78532 Tuttlingen, Germany; Allan.Maas@aesculap.de (A.M.); \\ svenja.ottawa@aesculap.de (S.O.); thomas.grupp@aesculap.de (T.M.G.) \\ 2 Department of Orthopaedic and Trauma Surgery, Musculoskeletal University Center Munich (MUM), \\ Campus Grosshadern, Ludwig Maximilians University Munich, 81377 Munich, Germany \\ 3 Faculty of Industrial Technologies, Campus Tuttlingen, University of Furtwangen, 78532 Tuttlingen, Germany \\ 4 Department of Orthopaedic Surgery, Medical University of Vienna, Waehringer Guertel 18-20, \\ 1090 Vienna, Austria; a.giurea@gmx.at \\ * Correspondence: Adrian.Sauer@aesculap.de
}

Citation: Sauer, A.; Maas, A.; Ottawa, S.; Giurea, A.; Grupp, T.M. Towards a New, Pre-Clinical, Subject-Independent Test Model for Kinematic Analysis after Total Knee Arthroplasty-Influence of the Proximo-Distal Patella Position and Patellar Tendon Stiffness. Appl. Sci. 2021, 11, 10322. https://doi.org/ 10.3390/app112110322

Academic Editor:

Cheng-Kung Cheng

Received: 30 September 2021 Accepted: 1 November 2021 Published: 3 November 2021

Publisher's Note: MDPI stays neutral with regard to jurisdictional claims in published maps and institutional affiliations.

Copyright: (c) 2021 by the authors. Licensee MDPI, Basel, Switzerland. This article is an open access article distributed under the terms and conditions of the Creative Commons Attribution (CC BY) license (https:/ / creativecommons.org/licenses/by/ $4.0 /)$.
Featured Application: This new framework for simulating the knee after total knee arthroplasty is intended to facilitate kinematic analyses in implant development processes and analyses of effects of various individual anatomic properties.

Abstract: Although simulation models are heavily used in biomechanical research and testing of TKA implants, pre-clinical tools for a holistic estimation of implant performance under dynamic loading conditions are rare. The objective of this study was the development of an efficient pre-clinical test method for analyzing knee contact mechanics and kinematics based on a dynamic FE model and to evaluate the effects of the proximo-distal patella position and the patellar tendon stiffness on the patellar kinematics. A finite element-based workflow for knee prostheses designs was developed based on standardized in vivo load data, which included the tibial forces and moments. In a new research approach, the tibial forces are used as input for the model, whereas the tibial moments were used to validate the results. For the standardized sit down, stand up, and knee bend load cycles, the calculated tibial moments show only small deviations from the reference values-especially for high flexion angles. For the knee bend cycle, the maximum absolute value of patellar flexion decreases for higher patellar tendon stiffness and more distally placed patellar components. Therefore, patellarelated clinical problems caused by patella baja may also arise if the patellar tendon is too weak for high tibiofemoral flexion angles.

Keywords: dynamic finite element model; knee kinematics; patellofemoral kinematics; pre-clinical testing; total knee arthroplasty; patellar tendon; patella alta; patella baja

\section{Introduction}

Total knee arthroplasty (TKA) is a prevalent treatment option for osteoarthritis [1] which is the indication for $88 \%$ of TKA surgeries [2]. Ten years after surgery, survival rates are $86.1-93.8 \%$ [3-8] and satisfaction rates are $81 \%$. This means $19 \%$ of patients are either dissatisfied with or uncertain about the results of their procedure [9]. The rate of revisions due to aseptic causes in the first 15 years after primary TKA is 3\% [10].

The detection and elimination of implant-related issues that may lead to clinical problems once the implant system is marketed is the main focus of the pre-clinical testing phase. Aside from continuous biomechanical research, there are additional mandatory steps for implant manufacturers. To achieve regulatory clearance, standardized tests need 
to be performed in the pre-clinical testing stage. The majority of these tests focus on single components under high cyclic [11-13] or static [14] loading conditions. Pre-clinical test methods under physiological loading conditions (e.g., simulation of daily living activities in a tribological wear simulator [15-19]) exist and enable more complex assessments, such as the analysis of the resulting knee kinematics with an implant.

However, running these tests is time-consuming and cost-intensive [20]. Hence, they are usually only executed towards the end of the design process, with only the final design or with very few design variants in a single size combination.

To analyze knee kinematics and possible biomechanical causes for unmet patient expectations after TKA, computational simulation of joint mechanics is frequently used [21-26]. The state of the art in biomechanical research to precisely predict the kinematics for one specific combination of implant design and subject are musculoskeletal models [27]. However, the great effort needed to adapt such musculoskeletal models to several design variants or size combinations prevents their routine use in early stages of implant design. On the other hand, there are only a few FE models published that are suitable for kinematic analyses [28,29].

To the best of our knowledge, there are no models available with the ability to compare multifarious implant designs and size combinations concerning kinematics and implant loads without massive effort for every single variation. With such a model, it would be possible to identify the combination of design parameters which leads to the best possible fit to the defined requirements [30].

This work is part of a project which aims to address this lack of dynamic FE models of the knee joint for design stage and pre-clinical testing of new prostheses. The newly developed model is intended to be a tool to compare implant loading, component stresses, and kinematic behavior of the tibiofemoral and the patellofemoral joint for various implant design variants or for variations of other parameters, such as the stiffness of the patellar tendon or any other ligaments. In this model, only standardized tibial loads and flexion angles [31] are needed as an input, and the relative kinematics are part of the results.

To be able to run meaningful parametrical studies to improve implant designs, the model should be fast to solve without compromising the informative value of the dynamic results. In accordance with all standardized testing scenarios, the influence of effects caused by individual patient parameters needs to be restrained in order to achieve comparable results.

Besides the possibility to analyze effects of implant design changes, this model is also intended to uncover effects of changes in the explicitly modeled anatomical structures. This will be shown within this study by analyzing the effects of varying patellar tendon stiffness and initial proximo-distal position of the patella on patellar kinematics.

\section{Materials and Methods}

Standardized loads from eight subjects undergoing total knee arthroplasty with instrumented implants were used as basis for this analysis. These loads are scaled to a bodyweight (BW) of $100 \mathrm{~kg}$ [31,32]. The three tibial force and moment components are given in a tibia-fixed coordinate system. In the simulation, the same coordinates are used. The $x-, y-$, and $z$-axes are directed laterally, anteriorly, and proximally, respectively, and the origin is placed halfway between the deepest points of the gliding surface of the polyethylene (PE) insert (Figure 1). 


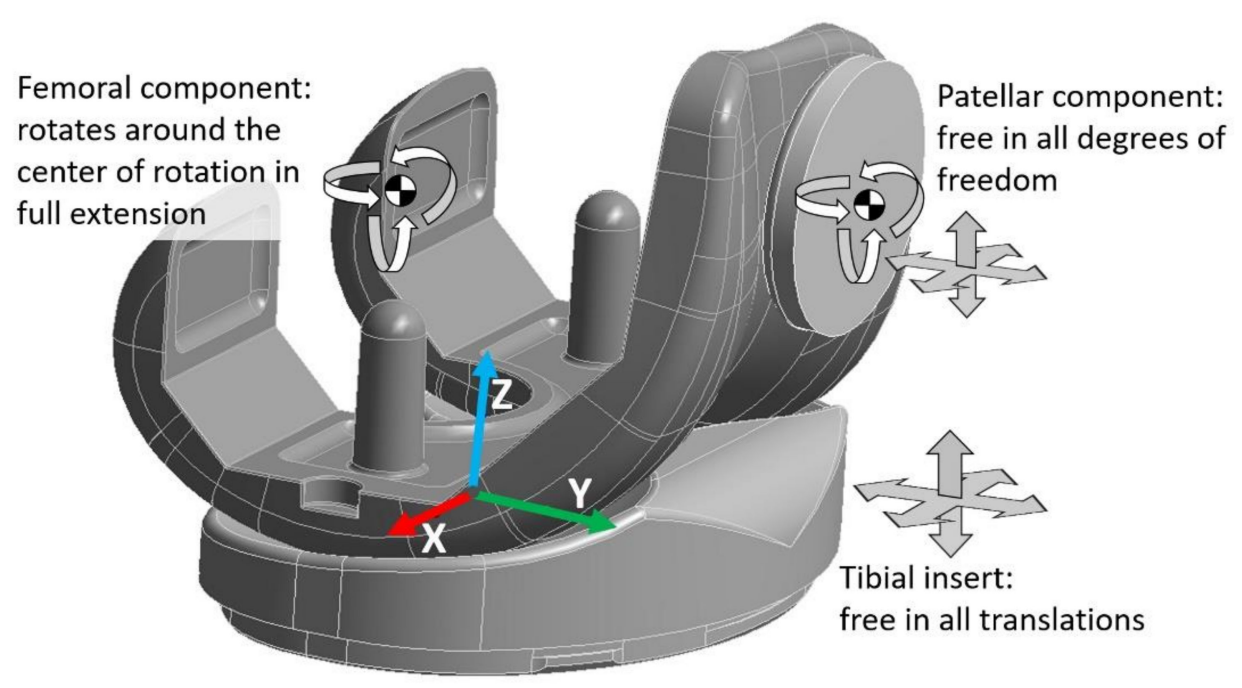

Figure 1. CAD model of the used implant components and the global coordinate system.

\subsection{Concept and Assumptions}

The central hypothesis for this model is that, in combination with the given tibial forces, only the real knee joint kinematics, especially the correct tibiofemoral contact situation, will lead to the given tibial reaction moment. Only the tibial forces and the flexion angle given by the dataset were used as input data to enable the validation of the FE-simulation results. The corresponding moments are then compared to the reaction moments output by the simulation.

In order to take the patella into account, the model must be adjusted such that the proportion of tibial forces caused by ground reaction forces are separated from the forces caused by muscles and ligaments. Without additional information regarding the direction and magnitude of ground reaction forces, it is not possible to achieve this reliably for load cycles with highly dynamic ground reaction forces. Therefore, two fundamentally different models were developed. The first includes the patella for the knee bend, sit down, and stand up cycles, and the second can handle all load cycles but does not contain any patella-related information. This paper presents only the first model, which includes the patella.

By using the standardized loads given by Bergmann et al. [31], patient-individual effects were reduced. These patient-individual effects were further reduced by avoiding individual bone geometries in the model. In this simulation, there was no need to consider bones and most muscles and ligaments because their impact is implied by the load data. Bergmann et al. [31] used the Zimmer Innex system (Zimmer Biomet, Warsaw, Indiana) for their measurements, a highly congruent, cruciate-sacrificing, and fixed-bearing implant. The properties of fixed-bearing tibial components allow the tibial implant component to be replaced by boundary conditions for the PE insert. Therefore, this model was reduced to the femoral component, the PE insert, and the patella component (Figure 1). Because the original implant geometry used in the study of Bergmann et al. [31] was not available, a comparable design (Columbus UC, Aesculap AG, Tuttlingen, Germany) and size combination (PE insert size 4, femur size 4, and patella size 1 ) was used.

\subsection{FE Model}

The six degrees of freedom between femoral and tibial implant component were defined as follows (Figure 1): The femoral component was free to revolve around a fixed center of rotation, which coincided with the center of rotation in full extension. The tibial component was free to translate in all axes with fixed rotations. The separation of translational and rotational degrees of freedom of the tibiofemoral joint in the described way led to a stable FE model without restricting the relative kinematics in any way. The flexion 
angle was the only degree of freedom, which was predefined by the dataset. So, there remain five degrees of freedom according to the tibiofemoral kinematics. The patella has six additional degrees of freedom and is only guided by its contact to the trochlear groove and four spring-elements, which modeled the function of the patellar and quadriceps tendons (Figures 1 and 2).

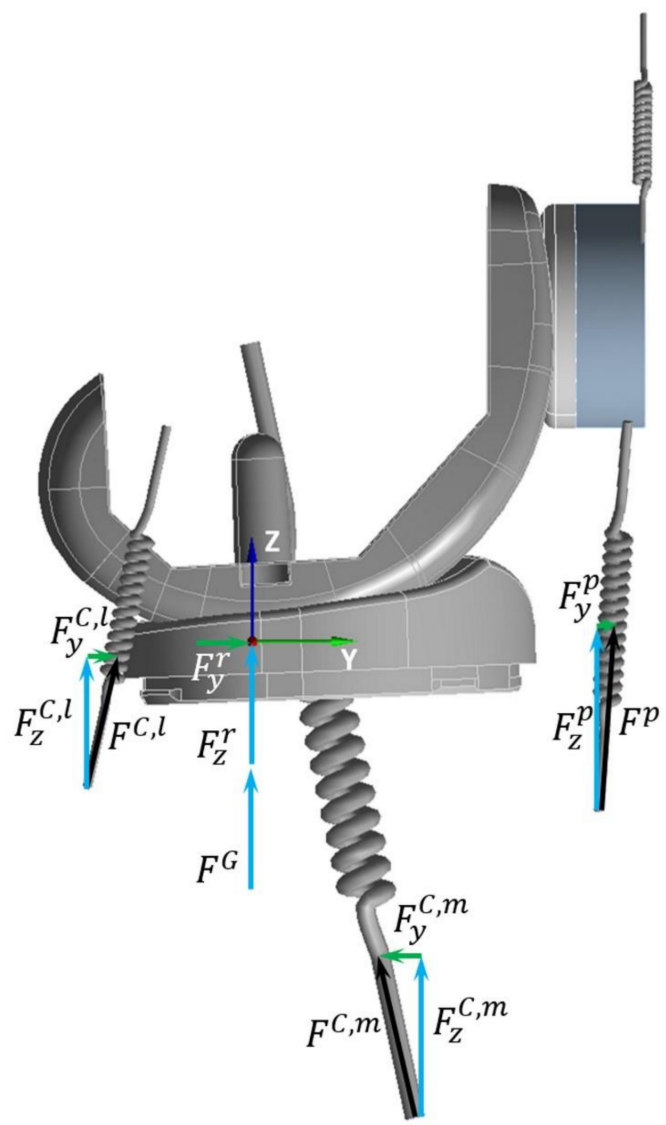

Figure 2. Tibial forces (black) and their components in $y$ (green)- and $z$ (blue)-directions, respectively.

There are two main contact areas in the knee joint which had to be taken into account. Both the tibiofemoral and the patellofemoral contact areas were modeled as asymmetric augmented Lagrangian contacts. A coefficient of friction of 0.05 was used for this simulation [33-35].

To minimize computing time, the small deformation of the femoral component was neglected, and it was instead defined as rigid body. Therefore, only some contact elements on its surface were needed, instead of a fully meshed geometry [36-38]. The material properties of the PE insert and the patella component were defined by a bilinear stressstrain curve with a yield strength of $25 \mathrm{MPa}$ [33].

In the first step of the calculation, the flexion angle and the standardized tibial forces $F_{x}^{B}, F_{y}^{B}$, and $F_{z}^{B}$ in the lateral ( $x$-axis), anterior ( $y$-axis), and proximal (z-axis) directions, as given by Bergmann et al. [31], were used as time-dependent input. Secondary input data were calculated from this primary data and provided as input for the FE model. The flexion angle was the only degree of freedom which was externally controlled. The given tibial forces could not directly be applied to the PE insert because the forces acting on the patellar tendon $\left(F^{P}\right)$ and collateral ligaments (medial: $F^{C, m}$ and lateral: $\left.F^{C, l}\right)$ and the ground reaction forces $\left(F^{G}\right)$ had to be subtracted first. In this study, the upper indices identified the forces or moments, while lower indices specified components of vectorial variables.

Forces caused by tissues, muscles, and ligaments, which are not explicitly modeled, are summarized by an additional force vector $F^{r}$. It complements the explicit modeled forces acting on the tibia to the standardized tibial loads $F^{B}$. This simplification allows 
to avoid the modeling of every single muscle and ligament, which would exceed the acceptable effort in modeling and solving for an everyday design evaluation tool without providing better results regarding implant loads and kinematics.

For the ground reaction force, $490.5 \mathrm{~N}$ (100 kg BW divided by two legs) are applied normal to the plane of the tibial plateau. Figure 2 shows the described forces with their components in the $y$ - and $z$-directions. In a frontal view, the same could be performed for the $x$ - and $z$-directions.

To split the standardized loads into the single forces stated above, the equilibrium of forces in the proximal $(z)$ and anterior $(y)$ directions from a sagittal view is set up. The described forces must replace the forces from the load data, which led to

$$
\begin{gathered}
F_{z}^{B}=F^{G}+F_{z}^{r}+F_{z}^{P}+F_{z}^{C, m}+F_{z}^{C, l} \text { and } \\
F_{y}^{B}=F_{y}^{r}+F_{y}^{P}+F_{y}^{C, m}+F_{y}^{C, l}
\end{gathered}
$$

The angle of the patellar tendon is used to derive the equation for $F_{y}^{P}$ from $F_{z}^{P}$, which results from Equation (1). Finally, Equation (2) gives the residual forces in the anterior direction. The described precalculation in the sagittal plane only considered forces acting parallel to this plane.

The forces of the collateral ligaments are initialized using their positions at full extension. Its $z$-components are calculated under the assumption that the patellar tendon force is zero. Therefore, an initial force distribution of $55 \%$ medial and $45 \%$ lateral is used, based on their ratio of ultimate tensile strength [39]. As shown in Figure 3, the same equation could be used to calculate the $z$-component of the patella tendon force for subsequent time steps by using the collateral ligament forces $F^{C, m}$ and $F^{C, l}$ calculated from kinematic results of previous simulation runs. By using the angle of the patellar tendon given by previous simulation runs, the total patellar-tendon force could be obtained, as well as its $y$-component, which is needed to calculate $F_{y}^{r}$ using Equation (2). The calculation of $F_{x}^{r}$ was analogous to the described algorithm for $F_{y}^{r}$.

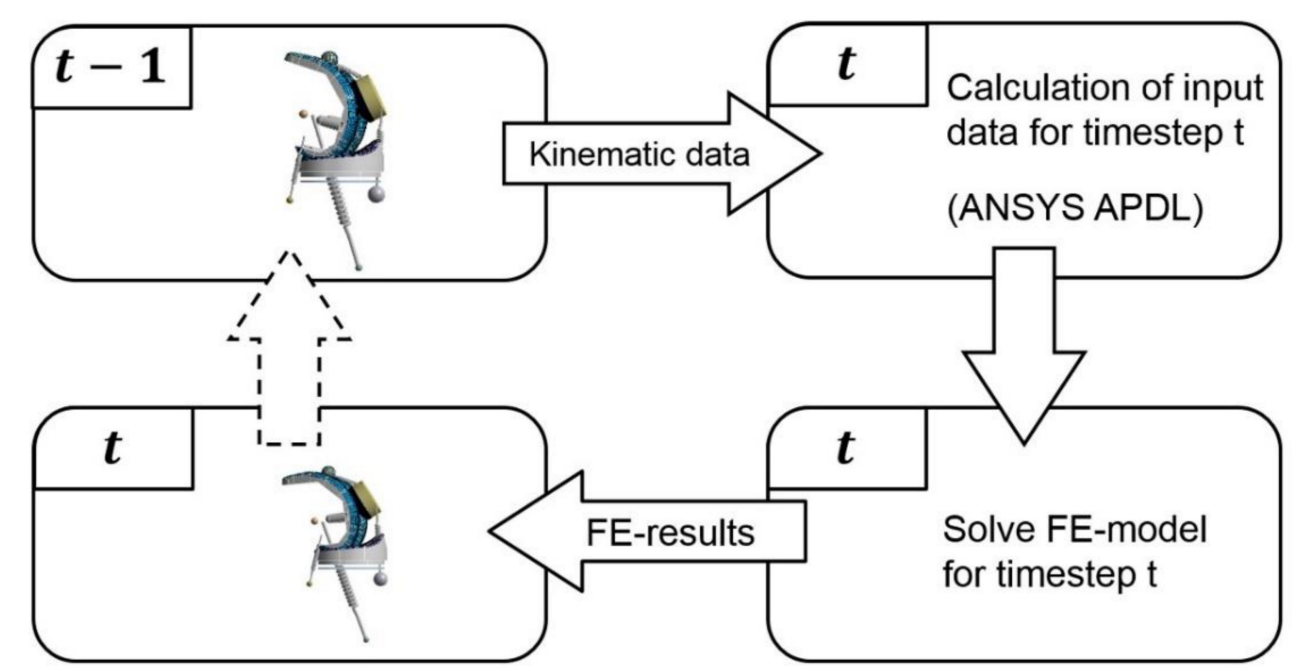

Figure 3. Iterative solving process using kinematic data from previous time step.

As per Wilson et al. [39], who reported no significant difference between medial and lateral collateral ligament, the stiffness of the collateral ligaments was set to $60 \mathrm{~N} / \mathrm{mm}$. The four studies in this review, which used moderate elongation rates $(\leq 200 \mathrm{~mm} / \mathrm{min})$, reported an averaged stiffness between 58 and $65 \mathrm{~N} / \mathrm{mm}$ [40-43].

To be able to consider the forces of the collateral ligaments when calculating the input data for the FE model, it was necessary to calculate their length using kinematic data from previous results. Therefore, the simulation was divided into 600 steps, one for every 
period between two data points in the given dataset from Bergmann et al. [31]. To calculate the input data $\left(F_{x}^{r}, F_{y}^{r}\right.$, and $\left.F_{z}^{r}\right)$ for time step $t$, the kinematic results from step $t-1$ were used. The deviation in tibial forces, which was caused by the delay of one time step of the kinematic data for calculating the input data, was negligible.

To implement the described algorithm, Ansys Workbench (Release 19.2) and Ansys Mechanical APDL were used. Inertial forces of the simulated components have only minor effects on this analysis because the simulated load cycles do not contain high accelerations and can be assumed as quasi-static. The small inertial forces of the body are implicitly included by using input data from instrumented implants. Nevertheless, to achieve a high numerical stability, a full transient analysis type using a sparse matrix direct solver was chosen in this study.

The tibial moments are given within the reference frame shown in Figure 1, and the tibiofemoral kinematics given in this study are defined as follows: the flexion angle is given as relative rotation of the femoral to the tibial component around the femoral medio-lateral axis of the implant. A positive femoral anterior-posterior translation (AP translation) is given as femoral movement to posterior parallel to the tibial $y$-axis, which is shown in Figure 1. The femoral rotation is the rotation of the femoral component around the tibial proximo-distal axis. Medial rotation is defined to be positive.

The patellar kinematics are given as relative movements of the patella with respect to the femoral coordinate system, as shown in Figure 4. The rotations are calculated as cardan sequence using the rotation order flexion-spin-tilt. Therefore, the patellar flexion is given as the relative rotation of the patella with respect to the femur around the femoral flexion axis. The values are negative if the patella flexes less than the femur, which is usually the case. The patellar tilt is described as the rotation around the patellar proximo-distal axis, with a positive sign if the patella rotates towards the medial side. For patellar spin, the axis is given as floating axis orthogonal to the other two. If the patellar spin is positive, then the proximal tip of the patella rotates towards the lateral side.

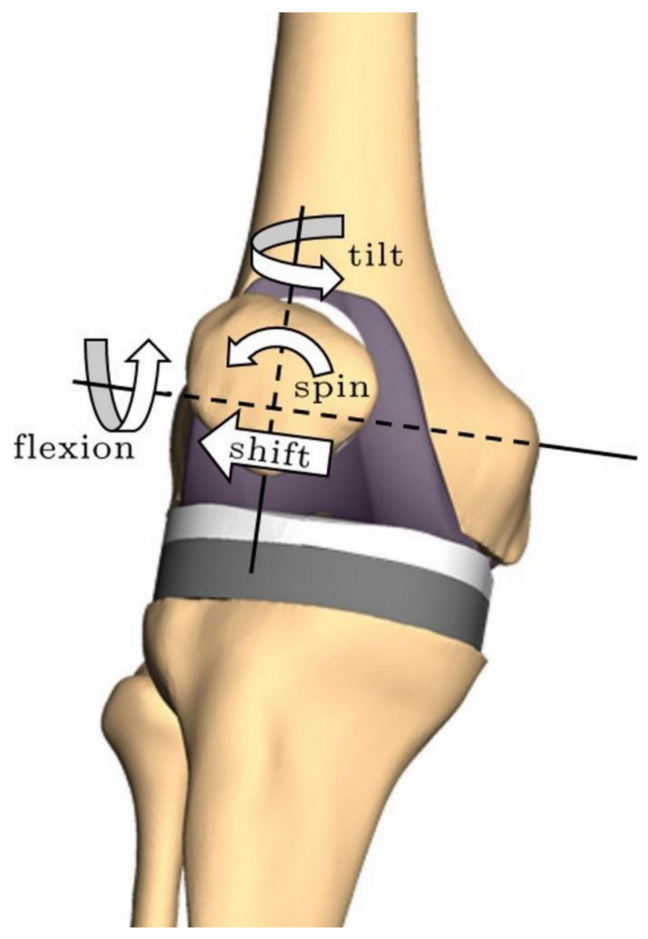

Figure 4. Patellar rotations and patellar shift. Shift along, and flexion around, femoral flexion axis, tilt around patellar proximo-distal axis, and spin around a floating axis orthogonal to the first two axes. Arrows are pointing to positive directions (basic image for this figure from model presented in [27]). 
A positive patellar shift is given as relative movement of the center point of the patella with respect to the femur in the lateral direction along the femoral flexion axis. All kinematics data are measured relative to their neutral position in full extension.

\section{Results}

The tibial reaction moments are the main results for the validation of the described model. The comparison between simulation results and the reference values from literature is shown in Figure 5 for the load cycles corresponding to knee bend, sit down, and stand up.

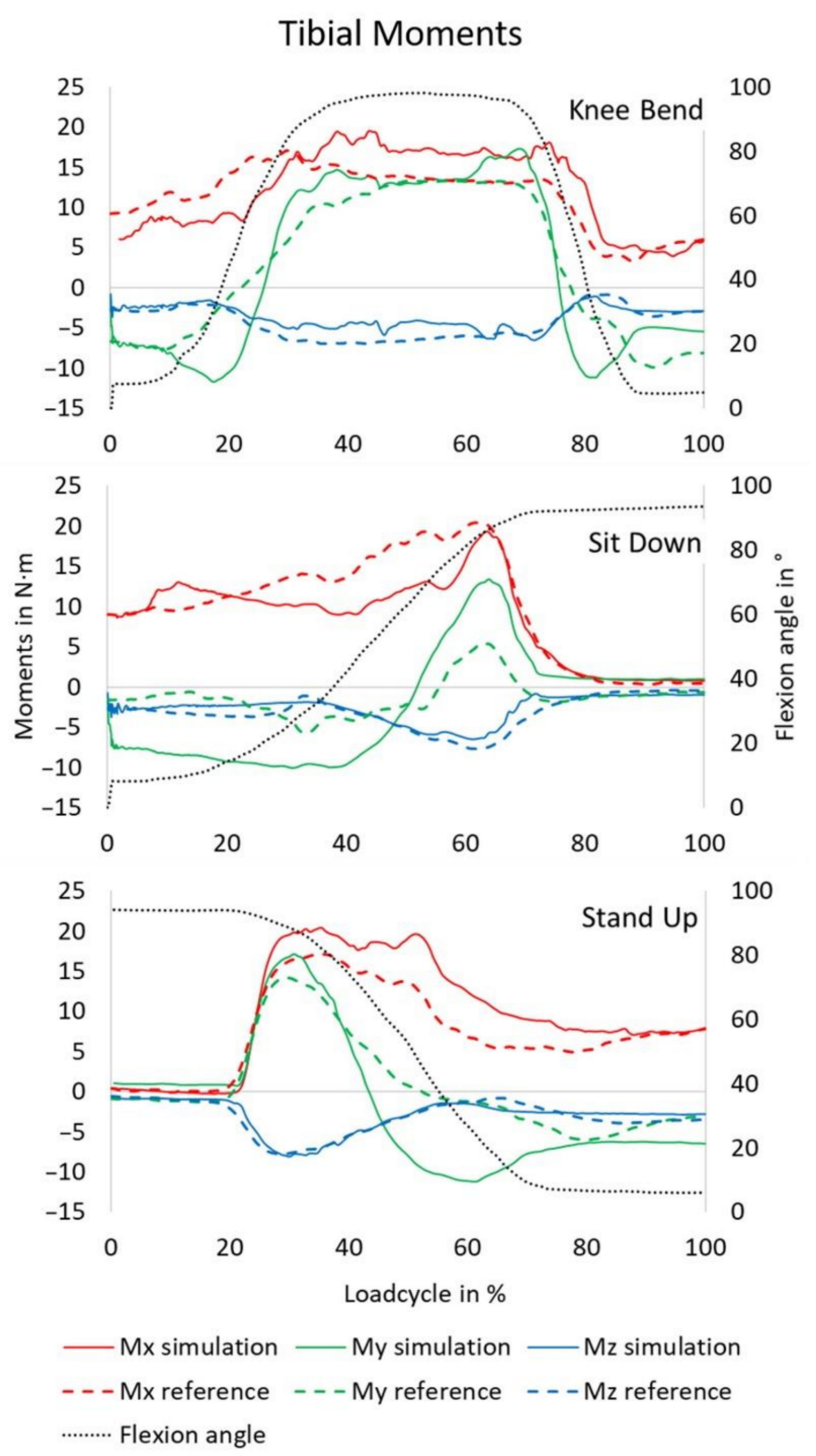

Figure 5. Comparison of simulation output to the reference data for the tibial moments for knee bend, sit down, and stand up cycles.

During weight-bearing squat, the medial tibial contact force was higher than the lateral one for low flexion angles. They showed equal values of $1350 \mathrm{~N}$ at the point of the zero-crossing of the varus-valgus moment at $75^{\circ}$ of flexion. For higher flexion angles, the lateral contact force was the dominant one. At $30^{\circ}\left(60^{\circ}, 90^{\circ}\right)$ of flexion, the medial and lateral tibial contact forces reached $999 \mathrm{~N}(1310 \mathrm{~N}, 1373 \mathrm{~N})$ and $478 \mathrm{~N}(950 \mathrm{~N}, 1700 \mathrm{~N})$. 
At the point of the maximum resultant tibial contact forces, the proportion of the medial contact force was $40 \%$ of the total tibial contact forces. Figure 6 shows the medial and lateral tibial contact forces for all three load cycles.
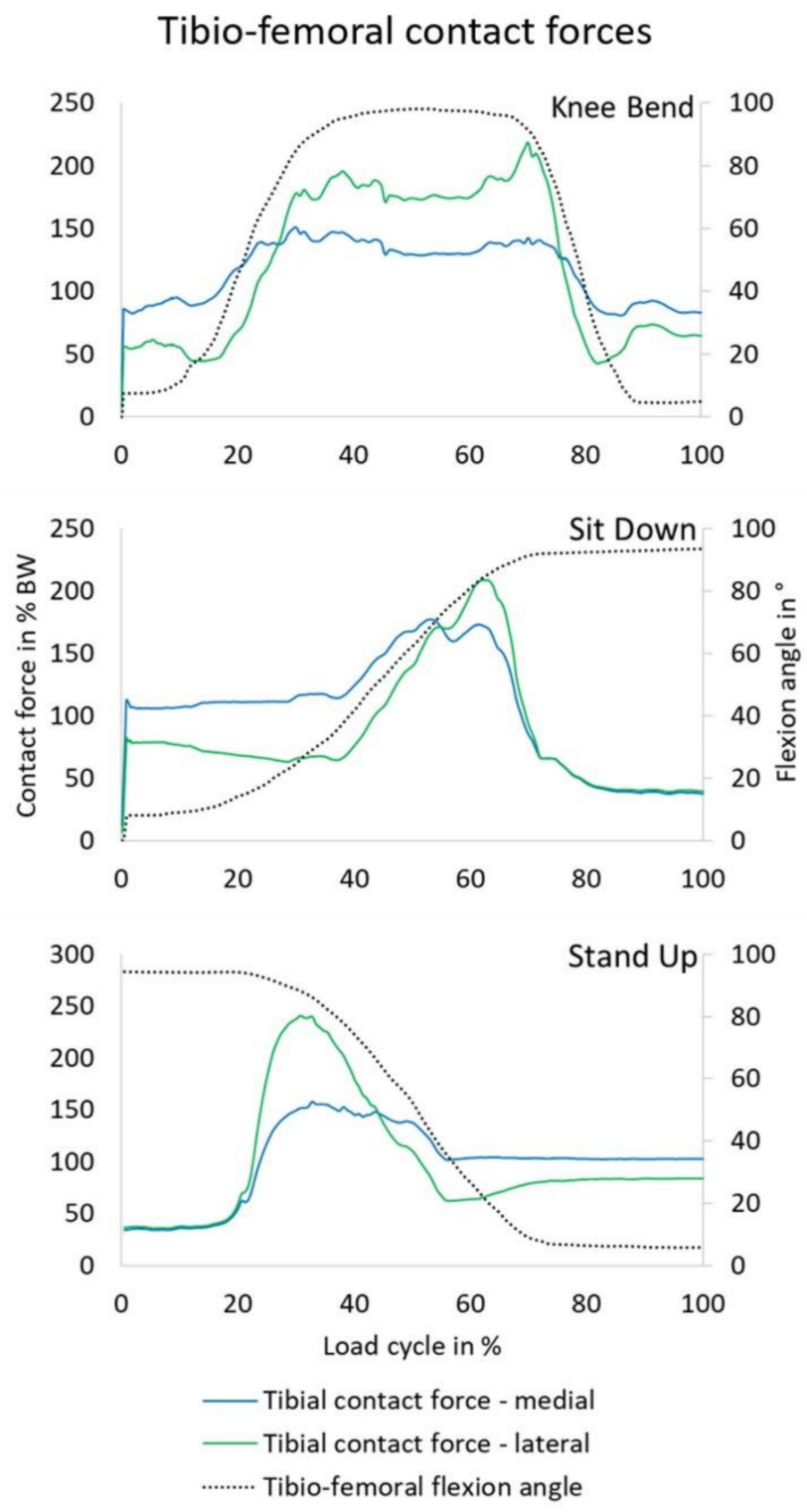

Figure 6. Tibial contact forces in medial and lateral compartment during weight-bearing squat.

For the knee bend cycle, the peak patellofemoral force was reached during the upwards movement at $91.4^{\circ}$ of flexion with a magnitude of $2563 \mathrm{~N}$, which is equal to $2.61 \mathrm{BW}$. The sit down cycle (stand up cycle) led to a maximum patellofemoral force of $2600 \mathrm{~N} / 2.65 \mathrm{BW}$ $(2872 \mathrm{~N} / 2.93 \mathrm{BW})$ at $84.2^{\circ}\left(86.1^{\circ}\right)$ of flexion.

The main translational and rotational degrees of freedom, which are femoral AP translation and tibial rotation around its axis, were analyzed and are shown in Figure 7. The mean relative femoral AP translation was equal to the tibial translation in the opposite direction, which was directly given by the model. For the $98^{\circ}$ squat, the maximum AP translation between femur and tibia was $16 \mathrm{~mm}$ at a flexion angle of $98.1^{\circ}$. 
Tibio-femoral Kinematics
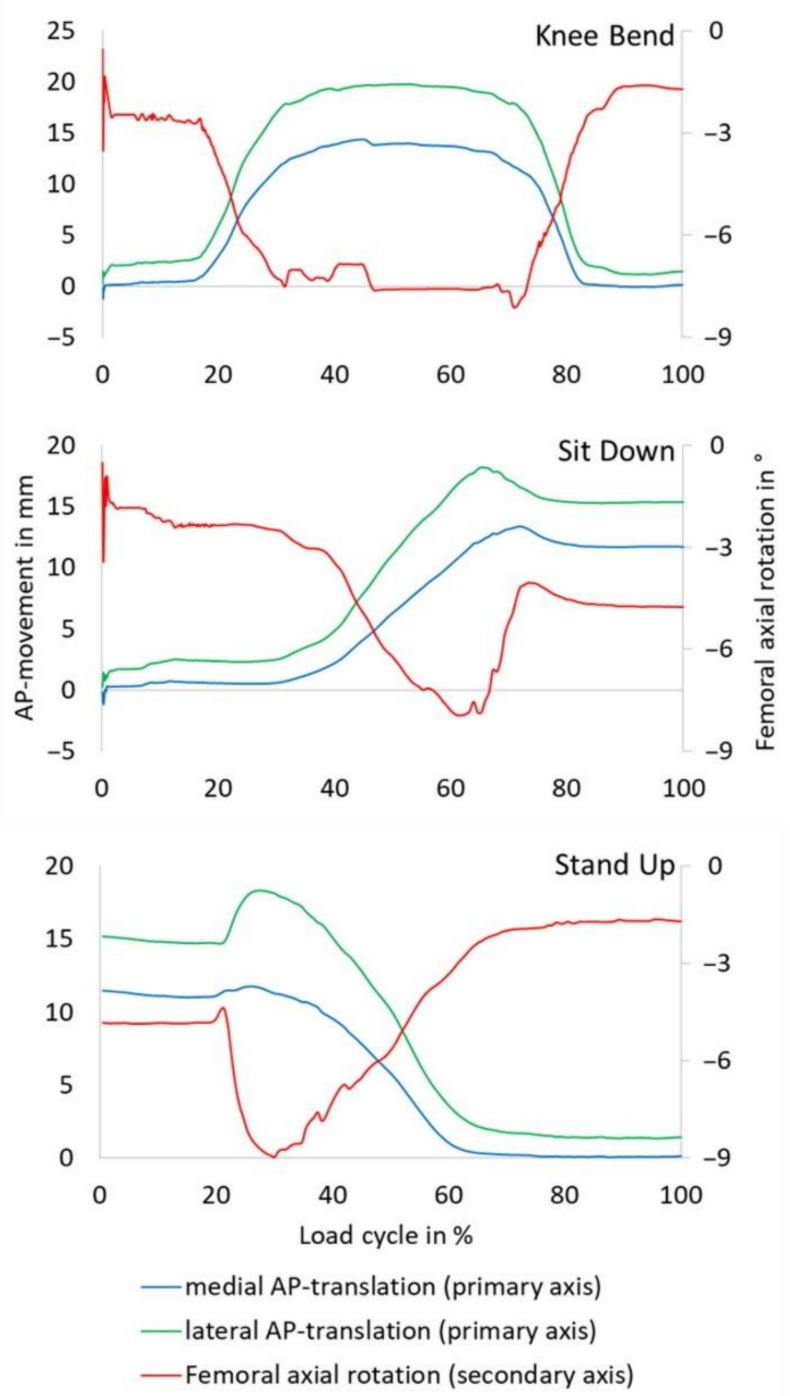

Figure 7. Femoral rollback of medial and lateral condyle and the axial femoral rotation for sit down, stand up, and knee bend cycle.

The tibial rotation could also be directly derived from this model by taking the femoral rotation around the tibial $z$-axis with a negative sign (Figure 7). A maximum tibial rotation of $8^{\circ}$ was calculated at $93.6^{\circ}$ of flexion (knee bend cycle).

For applications without evaluation of the tibiofemoral contact, the PE insert could be simulated as a rigid body to decrease computational time by about $10 \%$ without changing kinematics in a considerable way. The averaged difference between the curves of the tibial reaction moments was less than $4 \%$. Furthermore, taking peak patellofemoral force during the knee bend cycle as an example, this value changed by only $42 \mathrm{~N}$, going from $2539 \mathrm{~N}$ to $2581 \mathrm{~N}$ when simulating the PE insert as a rigid body.

The patellar kinematics were analyzed under variations of the patellar tendon stiffness between $300 \mathrm{~N} / \mathrm{mm}$ and $1000 \mathrm{~N} / \mathrm{mm}$, and the initial proximo-distal patellar position was simultaneously varied in a range of $\pm 6 \mathrm{~mm}$.

The peak values of patellar flexion, spin, and tilt during the downwards movement of the knee bend are given in Figures 8-10. The maximum absolute values of patellar flexion decrease for a decreasing stiffness of the patellar tendon and for a more proximally-placed patella. The peak values of patellar spin are between $3.64^{\circ}$ and $5.26^{\circ}$ of lateral rotation. 
The patellar spin increases with a weaker patellar tendon and for patellae placed more proximally. The range of peak values of patellar tilt is between $3.35^{\circ}$ and $3.68^{\circ}$ of medial tilt and no clear relationship to the initial patellar proximo-distal position or the stiffness of the patellar tendon can be seen.

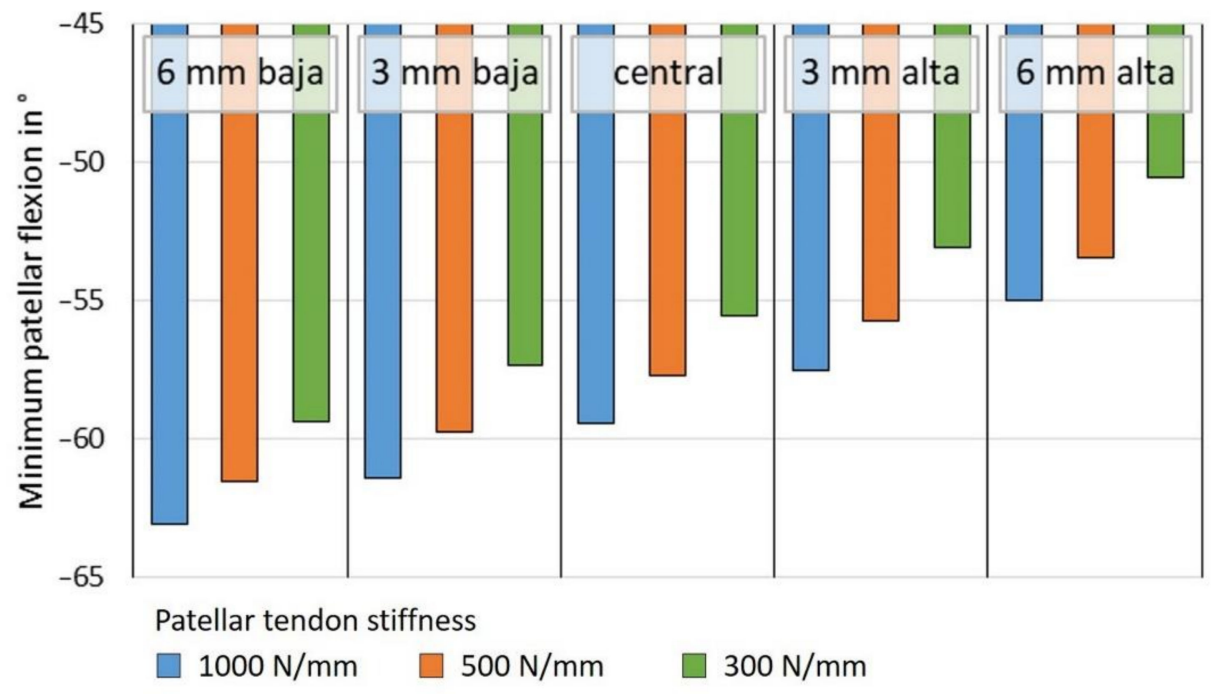

Figure 8. Peak values of the patellar flexion during weight-bearing squat for several initial patellar positions (left to right: $6 \mathrm{~mm}$ baja to $6 \mathrm{~mm}$ alta) and patellar tendon stiffnesses (blue: $1000 \mathrm{~N} / \mathrm{mm}$, orange: $500 \mathrm{~N} / \mathrm{mm}$, and green: $300 \mathrm{~N} / \mathrm{mm}$ ). Flexion is given around the femoral flexion axis relative to the femoral implant component. Negative values indicate that the patella flexes less than the femur.

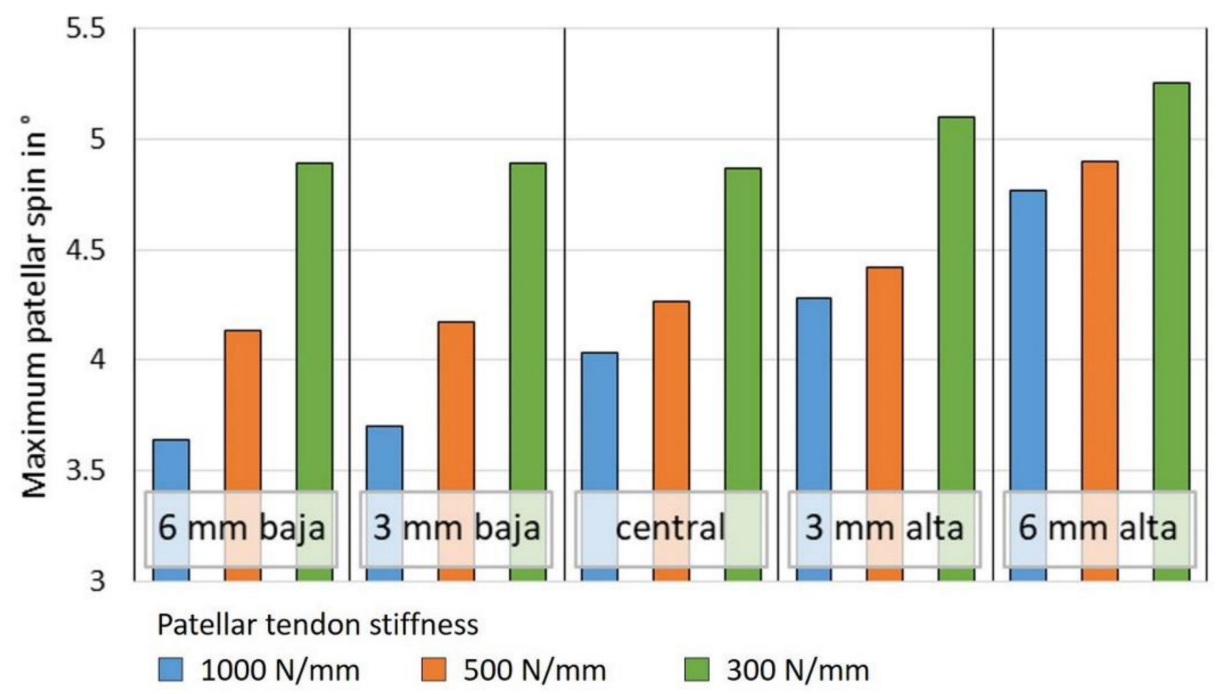

Figure 9. Peak values of the patellar spin during weight-bearing squat for several initial patellar positions (left to right: $6 \mathrm{~mm}$ baja to $6 \mathrm{~mm}$ alta) and patellar tendon stiffnesses (blue: $1000 \mathrm{~N} / \mathrm{mm}$, orange: $500 \mathrm{~N} / \mathrm{mm}$, and green: $300 \mathrm{~N} / \mathrm{mm}$ ). Spin is given as rotation around the floating axis between the femoral flexion axis and the patellar proximo-distal axis. A positive sign indicates a rotation to lateral direction. 


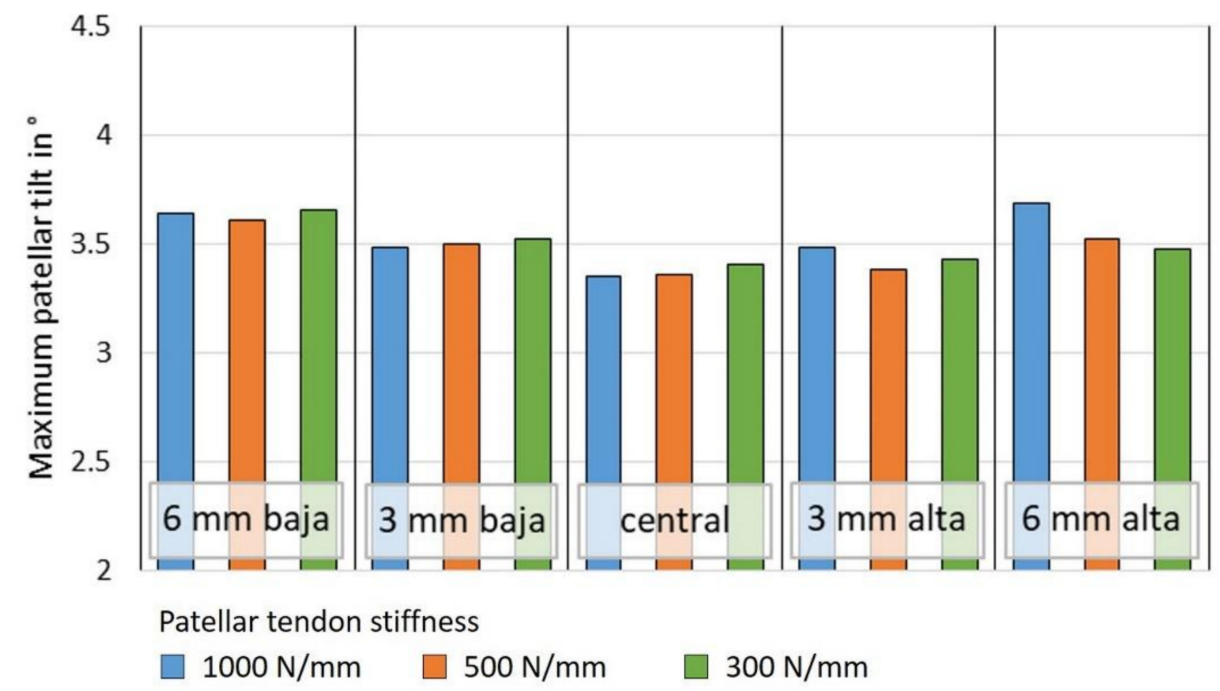

Figure 10. Peak values of the patellar tilt during weight-bearing squat for several initial patellar positions (left to right: $6 \mathrm{~mm}$ baja to $6 \mathrm{~mm}$ alta) and patellar tendon stiffnesses (blue: $1000 \mathrm{~N} / \mathrm{mm}$, orange: $500 \mathrm{~N} / \mathrm{mm}$, and green: $300 \mathrm{~N} / \mathrm{mm}$ ). Patellar tilt is given as rotation around the patellar proximo-distal axis. Rotations of the patella to the medial side have a positive sign.

The peaks of the patellar shift shown in Figure 11 are between $0.27 \mathrm{~mm}$ and $0.75 \mathrm{~mm}$ to the medial side. While the initial proximo-distal position of the patella influences the peak patellar shift, the stiffness of the patellar tendon has only minor effects on the patellar shift.

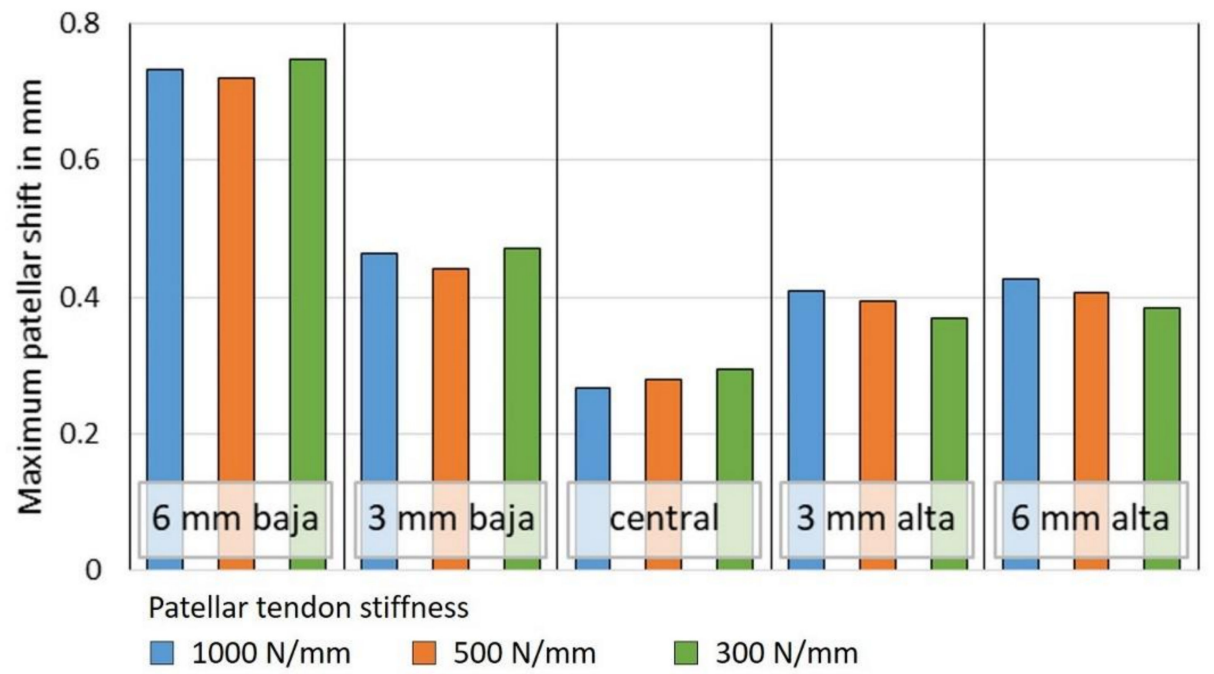

Figure 11. Peak values of the patellar shift during weight-bearing squat for several initial patellar positions (left to right: $6 \mathrm{~mm}$ baja to $6 \mathrm{~mm}$ alta) and patellar tendon stiffnesses (blue: $1000 \mathrm{~N} / \mathrm{mm}$, orange: $500 \mathrm{~N} / \mathrm{mm}$, and green: $300 \mathrm{~N} / \mathrm{mm}$ ). A positive patellar shift is given for relative patellar movements along the femoral mediolateral axis in lateral direction.

Depending on the question that is addressed with this framework, solution times vary from $50 \mathrm{~min}$ in a rigid body setup, to determine moment reactions and resulting kinematics, to $4 \mathrm{~h}$ when detailed results of the contact situation of a flexible PE component are needed with a high mesh density and element sizes down to $0.75 \mathrm{~mm}$.

\section{Discussion}

The objective of this study was to develop a dynamic FE model for pre-clinical analysis of knee contact mechanics and kinematics based on standardized loading conditions and to 
analyze the effects of various changes of anatomic properties of a knee, such as the patellar tendon stiffness or the proximo-distal position of the patella. This model was intended to be used to analyze multifarious design variants during the implant design and pre-clinical testing process using a fast and straightforward workflow. Therefore, it should be fast to adapt and solve.

The reduced complexity of the model was achieved by partially replacing tissues and bone geometries by boundary conditions. Therefore, the informative value of the results was limited to implant kinematics, loads, and stresses. Findings according to the specific behavior of soft tissue structures surrounding the knee were limited to the explicitly modeled structures, such as the patellar tendon. However, established pre-clinical testing methods are either based on nonstandardized data (e.g., [44,45]) or neglect soft tissue structures and other patient-specific parameters (e.g., [18]). Therefore, the limitation that only the effects of some soft tissue structures can be analyzed turns into an advantage relative to other established standardized pre-clinical testing methods.

Another limitation may arise by the fact that implant geometries were used, which were slightly different from the implants used to create the standardized loads [31]. However, Freed et al. [46] compared the axial tibial loads from measurements and a numeric model with the standardized loads, and even though they used a different ultra-congruent cruciate sacrificing implant than Bergmann et al. [31], there were no significant differences for the quasi-static load cycles of sitting down and rising from a chair. These load cycles are similar to the knee bend cycle, which was also used in our study. Therefore, it is assumed that the use of the implant components of Columbus ${ }^{\circledR} \mathrm{UC}$ did not cause substantial differences.

The remaining data from the given dataset, which were not used as input for the simulation, could be used to evaluate the simulation results. The moment around the flexion axis $\left(M_{x}\right)$ and the varus-valgus moment $\left(M_{y}\right)$ were in good accordance with deep flexion angles, and a deviation could be seen in extension. Regarding the moment around the tibial axis $\left(M_{z}\right)$, the simulation results were also consistent with the dataset from literature for small flexion angles. As shown in Figure 5, all of the curves show characteristics which are similar to the standardized load data. Therefore, the model seems to be reliable according to tibial moments and forces, especially for deeper flexion angles, which are more relevant and critical to simulate.

The contact forces in the tibial condyles were compared to an analysis of Kutzner et al. [47], who calculated the medial force ratios from nine patients for different daily activities. Some of their patients were also in the patient cohort for the standardized loads [31], which were used in the present study. If the different level of the total resulting forces is considered, the curves of medial and lateral tibial contact forces are similar for the present study compared to Kutzner et al. [47]. The medial force ratio in the tibia was $40 \%$ at the instant of maximum total contact forces, which fits well to the $42 \% \pm 8 \%$ which were given by Kutzner et al. [47]. The varus-valgus moment is changing its sign from negative to positive at $75^{\circ}$ of flexion during knee bend. This results in a switch of the dominant tibiofemoral contact force from the medial to the lateral condyle, as shown in Figure 6. Therefore, the most loaded condyle in a deep knee bend cycle is the lateral one. At first glance, this might be inconsistent with the fact that in most patients, the medial condyle is more affected by osteoarthritis than the lateral one [48] and 90-95\% of unicompartmental knee arthroplasties are placed on the medial side [49]. A deeper look into data from instrumented knee implants [31] shows positive varus-valgus moments mainly for deep flexion angles in symmetric two-legged load cycles, such as knee bend, sit down, and stand up from a chair. The lower relative prevalence of these cycles, compared to level walking, stairs up, and stairs down cycles, with high negative varus-valgus moments explain the higher risk of osteoarthritis on the medial condyle. For the sit down and stand up cycles, the results are also in good agreement with the given data from Kutzner et al. [47].

For two of the eight patients that were included into the standardized loads from Bergmann et al. [31], a subject-specific calculation was made by Trepczynski et al. [50]. 
They calculated 3.2 BW as averaged peak patellofemoral contact force during a squat at a flexion angle of $94^{\circ}$. Their results for the two patients were 2.6 BW and 3.7 BW. The maximum of $2.6 \mathrm{BW}$ for the patella contact force at $91.4^{\circ}$ of flexion that was detected in the present study is equal to the lower bound of the study stated above. Research from other groups, with different patient cohorts, showed patellofemoral forces for a squat at $100^{\circ}$ of flexion in a range of $2.7 \mathrm{BW}$ to $3.5 \mathrm{BW}$ [51,52].

Compared to the given literature values, the results of the herein described simulation approach show good agreement, especially for high flexion angles, where the most critical loads are reached. Therefore, the model demonstrates potential to be a useful tool to evaluate implant designs in a platform development approach of new knee systems. It is possible to fully automate the simulation process, which makes it possible to test a great number of geometries in an efficient way. To improve the model, additional validation steps can be performed in future with the CAMS-Knee dataset. This dataset contains tibial loads and kinematics data measured by video fluoroscopy [53].

Further development of this methodology can enable implant manufacturers to achieve more realistic kinematic behavior on a systematic scale by considering dynamic loading and tibio- and patellofemoral kinematics, as well as contact mechanics in a standardized workflow.

Especially in combination with a second model, which will be able to handle load cycles with dynamic ground reaction forces but dispenses with considering the patella, this method opens a wide field of subjects to investigate in an efficient way-from effects of design changes in patellar and trochlear geometries to the impact of different alignment methods.

The analysis of patellar kinematics shows the effects of proximo-distal patellar position and patellar tendon stiffness. According to patellar flexion, both patella baja and high patellar tendon stiffness lead to an increase in relative patellar flexion angles. Therefore, problems occurring in knees with patella baja for high flexion angles could also occur in knees with a central patellar position and a very strong patellar tendon. A weak patellar tendon also increases patellar spin and has minor effects on patellar tilt and shift. The proximo-distal position of the patella is the main parameter to determine the patellar tilt and shift. Both have their minimum for a central patella and increase for patella baja and alta, where the effect is stronger for the patella baja. The changes in the peak values for shift and tilt are very small, with a maximum difference of $0.27 \mathrm{~mm}$ and $0.33^{\circ}$. Therefore, the effects of the proximo-distal position of the patella and the patellar tendon stiffness on shift and tilt seem to have only minor clinical relevance.

A patella baja can lead to impingement, anterior knee pain, and a reduced range of motion $[54,55]$. Consequently, the described changes in patellar kinematics may have an impact on TKA performance and satisfaction in patients with patella baja.

\section{Conclusions}

A new approach for FE analysis of the knee contact mechanics and kinematics after TKA, which allows to compare multifarious design variants in an efficient way, is presented. As input data, standardized loads for knee bend, sit down, and stand up cycles were used. Some elements of these datasets were used to validate the model.

Due to the use of data based on measurements of instrumented tibial implants, only a few ligament and muscle forces had to be modeled explicitly. The remaining tissues were implicitly contained into the tibial forces used as input. This way of reducing the complexity of the model led to an easy to handle model, which can be solved fast. This model is suitable for the intended use of pre-clinical screening of many design variants, unlike many common pre-clinical testing models, which are mostly static and therefore not suitable to analyze kinematic properties of implant designs.

The presented model is additionally capable to analyze effects of various parameters, such as implant positions or properties of the explicitly modeled ligaments such as patellar tendon stiffness. By varying these two parameters, this study showed that changes of the 
patellar tendon stiffness have very similar effects on patellar flexion for high tibiofemoral flexion angles. Therefore, clinical issues related to patella baja or alta in deep tibiofemoral flexion could also be caused by changes in patellar tendon stiffness for normal proximodistal position of the patella in extension.

Author Contributions: Conceptualization, A.S., A.M. and T.M.G.; methodology, A.S., A.M. and S.O.; validation, A.S. and A.M.; formal analysis, A.S. and S.O.; resources, A.M. and T.M.G.; data curation, A.S.; investigation, A.S., A.M., S.O., A.G. and T.M.G.; writing—original draft preparation, A.S.; writing-review and editing, A.S., A.M., A.G. and T.M.G.; visualization, A.S.; supervision, A.M., A.G. and T.M.G.; project administration, A.M. and T.M.G.; funding acquisition, A.M. and T.M.G. All authors have read and agreed to the published version of the manuscript.

Funding: Four of the authors (A.S., A.M., S.O. and T.M.G.) were funded by B. Braun Aesculap A.G., Tuttlingen. The funder provided support in the form of salaries for authors A.S., A.M., S.O. and T.M.G. The funders had no role in study design, data collection and analysis, decision to publish, or preparation of the manuscript.

Institutional Review Board Statement: Not applicable.

Informed Consent Statement: Informed consent was obtained from all subjects involved in the study.

Data Availability Statement: The data presented in this study are available in article.

Acknowledgments: Special thanks to Maeruan Kebbach (Department of Orthopaedics, Rostock University Medical Center, Rostock, Germany) for providing the basic image for Figure 4 Ceramics in substitutive.

Conflicts of Interest: Four of the authors (A.S., A.M., S.O. and T.M.G.) are employees of B. Braun Aesculap A.G., Tuttlingen. A.G. is a paid consultant for presentations (B. Braun and De Puy) and receives royalties from $B$. Braun, but did not receive any reimbursement for the current study.

\section{References}

1. Rönn, K.; Reischl, N.; Gautier, E.; Jacobi, M. Current surgical treatment of knee osteoarthritis. Arthritis 2011, $2011,454873$. [CrossRef]

2. Robertsson, O.; Bizjajeva, S.; Fenstad, A.M.; Furnes, O.; Lidgren, L.; Mehnert, F.; Odgaard, A.; Pedersen, A.B.; Havelin, L.I. Knee arthroplasty in Denmark, Norway and Sweden. A pilot study from the Nordic Arthroplasty Register Association. Acta Orthop. 2010, 81, 82-89. [CrossRef]

3. Bae, D.K.; Song, S.J.; Heo, D.B.; Lee, S.H.; Song, W.J. Long-term survival rate of implants and modes of failure after revision total knee arthroplasty by a single surgeon. J. Arthroplast. 2013, 28, 1130-1134. [CrossRef]

4. Argenson, J.-N.; Boisgard, S.; Parratte, S.; Descamps, S.; Bercovy, M.; Bonnevialle, P.; Briard, J.-L.; Brilhault, J.; Chouteau, J.; Nizard, R.; et al. Survival analysis of total knee arthroplasty at a minimum 10 years' follow-up: A multicenter French nationwide study including 846 cases. Orthop. Traumatol. Surg. Res. 2013, 99, 385-390. [CrossRef]

5. Bae, D.K.; Song, S.J.; Park, M.J.; Eoh, J.H.; Song, J.H.; Park, C.H. Twenty-year survival analysis in total knee arthroplasty by a single surgeon. J. Arthroplast. 2012, 27, 1297-1304. [CrossRef] [PubMed]

6. Koskinen, E.; Eskelinen, A.; Paavolainen, P.; Pulkkinen, P.; Remes, V. Comparison of survival and cost-effectiveness between unicondylar arthroplasty and total knee arthroplasty in patients with primary osteoarthritis: A follow-up study of 50,493 knee replacements from the Finnish Arthroplasty Register. Acta Orthop. 2008, 79, 499-507. [CrossRef] [PubMed]

7. Labek, G.; Thaler, M.; Janda, W.; Agreiter, M.; Stöckl, B. Revision rates after total joint replacement: Cumulative results from worldwide joint register datasets. J. Bone Jt. Surg. Br. 2011, 93, 293-297. [CrossRef] [PubMed]

8. Sadoghi, P.; Liebensteiner, M.; Agreiter, M.; Leithner, A.; Böhler, N.; Labek, G. Revision surgery after total joint arthroplasty: A complication-based analysis using worldwide arthroplasty registers. J. Arthroplast. 2013, 28, 1329-1332. [CrossRef]

9. Robertsson, O.; Dunbar, M.; Pehrsson, T.; Knutson, K.; Lidgren, L. Patient satisfaction after knee arthroplasty: A report on 27,372 knees operated on between 1981 and 1995 in Sweden. Acta Orthop. Scand. 2000, 71, 262-267. [CrossRef]

10. Jorgensen, N.B.; McAuliffe, M.; Orschulok, T.; Lorimer, M.F.; de Steiger, R. Major Aseptic Revision Following Total Knee Replacement: A Study of 478,081 Total Knee Replacements from the Australian Orthopaedic Association National Joint Replacement Registry. J. Bone Jt. Surg. Am. 2019, 101, 302-310. [CrossRef]

11. ASTM F2723-13a. Standard Test Method for Evaluating Mobile Bearing Knee Tibial Baseplate/Bearing Resistance to Dynamic Disassociation; ASTM International: West Conshohocken, PA, USA, 2013.

12. ASTM F2722-15. Standard Practice for Evaluating Mobile Bearing Knee Tibial Baseplate Rotational Stops; ASTM International: West Conshohocken, PA, USA, 2015. 
13. ASTM F2777-16. Standard Test Method for Evaluating Knee Bearing (Tibial Insert) Endurance and Deformation Under High Flexion; ASTM International: West Conshohocken, PA, USA, 2016.

14. ASTM F2083-12. Standard Specification for Knee Replacement Prosthesis; ASTM International: West Conshohocken, PA, USA, 2012.

15. ISO 14243-1:2009-11. Implants for Surgery —Wear of Total Knee Prostheses_Part 1: Loading and Displacement Parameters for Wear-Testing Machines with Load Control and Corresponding Environmental Conditions for Test; ISO: Geneva, Switzerland, 2009.

16. ISO 14243-3_2014-11. Implants for Surgery_Wear of Total Knee Prostheses_Part 3: Loading and Displacement Parameters for WearTesting Machines with Displacement Control and Corresponding Environmental Conditions for Test; ISO: Geneva, Switzerland, 2009.

17. ASTM F3141-17a. Standard Guide for Total Knee Replacement Loading Profiles; ASTM International: West Conshohocken, PA, USA, 2017.

18. Schwiesau, J.; Schilling, C.; Kaddick, C.; Utzschneider, S.; Jansson, V.; Fritz, B.; Blömer, W.; Grupp, T.M. Definition and evaluation of testing scenarios for knee wear simulation under conditions of highly demanding daily activities. Med. Eng. Phys. 2013, 35, 591-600. [CrossRef]

19. ISO 14243-5:2019-05. Implants for Surgery—Wear of Total Knee Prostheses_Part 5: Durability Performance of the Patellofemoral Joint; ISO: Geneva, Switzerland, 2019.

20. Halloran, J.P.; Clary, C.W.; Maletsky, L.P.; Taylor, M.; Petrella, A.J.; Rullkoetter, P.J. Verification of Predicted Knee Replacement Kinematics During Simulated Gait in the Kansas Knee Simulator. J. Biomech. Eng. 2010, 132, 081010. [CrossRef] [PubMed]

21. Ardestani, M.M.; Moazen, M.; Maniei, E.; Jin, Z. Posterior stabilized versus cruciate retaining total knee arthroplasty designs: Conformity affects the performance reliability of the design over the patient population. Med. Eng. Phys. 2015, 37, 350-360. [CrossRef]

22. Chen, Z.; Jin, Z. Prediction of in-vivo kinematics and contact track of total knee arthroplasty during walking. Biosurf. Biotribol. 2016, 2, 86-94. [CrossRef]

23. Kang, K.-T.; Koh, Y.-G.; Son, J.; Kwon, O.-R.; Baek, C.; Jung, S.H.; Park, K.K. Measuring the effect of femoral malrotation on knee joint biomechanics for total knee arthroplasty using computational simulation. Bone Jt. Res. 2016, 5, 552-559. [CrossRef] [PubMed]

24. Osano, K.; Nagamine, R.; Todo, M.; Kawasaki, M. The effect of malrotation of tibial component of total knee arthroplasty on tibial insert during high flexion using a finite element analysis. Sci. World J. 2014, 2014, 695028. [CrossRef]

25. Steinbrück, A.; Woiczinski, M.; Weber, P.; Müller, P.E.; Jansson, V.; Schröder, C. Posterior cruciate ligament balancing in total knee arthroplasty: A numerical study with a dynamic force controlled knee model. Biomed. Eng. Online 2014, 13, 91. [CrossRef]

26. Taylor, M.; Barrett, D.S. Explicit finite element simulation of eccentric loading in total knee replacement. Clin. Orthop. Relat. Res. 2003, 414, 162-171. [CrossRef]

27. Kebbach, M.; Darowski, M.; Krueger, S.; Schilling, C.; Grupp, T.M.; Bader, R.; Geier, A. Musculoskeletal Multibody Simulation Analysis on the Impact of Patellar Component Design and Positioning on Joint Dynamics after Unconstrained Total Knee Arthroplasty. Materials 2020, 13, 2365. [CrossRef] [PubMed]

28. Kang, K.-T.; Koh, Y.-G.; Son, J.; Kwon, O.-R.; Lee, J.-S.; Kwon, S.-K. Influence of Increased Posterior Tibial Slope in Total Knee Arthroplasty on Knee Joint Biomechanics: A Computational Simulation Study. J. Arthroplast. 2018, 33, 572-579. [CrossRef]

29. Woiczinski, M.; Steinbruck, A.; Weber, P.; Muller, P.E.; Jansson, V.; Schröder, C. Development and validation of a weight-bearing finite element model for total knee replacement. Comput. Methods Biomech. Biomed. Eng. 2016, 19, 1033-1045. [CrossRef]

30. Rullkoetter, P.J.; Fitzpatrick, C.K.; Clary, C.W. How Can We Use Computational Modeling to Improve Total Knee Arthroplasty? Modeling Stability and Mobility in the Implanted Knee. J. Am. Acad. Orthop. Surg. 2017, 25 (Suppl. 1), S33-SS39. [CrossRef]

31. Bergmann, G.; Bender, A.; Graichen, F.; Dymke, J.; Rohlmann, A.; Trepczynski, A.; Heller, M.O.; Kutzner, I. Standardized loads acting in knee implants. PLoS ONE 2014, 9, e86035. [CrossRef]

32. Heinlein, B.; Graichen, F.; Bender, A.; Rohlmann, A.; Bergmann, G. Design, calibration and pre-clinical testing of an instrumented tibial tray. J. Biomech. 2007, 40 (Suppl. 1), S4-S10. [CrossRef] [PubMed]

33. Maas, A.; Kim, T.K.; Miehlke, R.K.; Hagen, T.; Grupp, T.M. Differences in anatomy and kinematics in Asian and Caucasian TKA patients.: Influence on implant positioning and subsequent loading conditions in mobile bearing knees. BioMed Res. Int. 2014, 2014, 612838. [CrossRef]

34. Yao, J.Q.; Laurent, M.P.; Johnson, T.S.; Blanchard, C.R.; Crowninshield, R.D. The influences of lubricant and material on polymer/CoCr sliding friction. Wear 2003, 255, 780-784. [CrossRef]

35. Fricker, D.C. Friction when femoral prosthesis heads slide in acetabular cups in Ceramics in substitutive and reconstructive surgery. In Proceedings of the Satellite Symposium 3 on Ceramics in Substitutive and Reconstructive Surgery, Montecatini Terme, Italy, 27-30 June 1990; Elsevier: New York, NY, USA, 1991; pp. 207-215.

36. D'Lima, D.D.; Chen, P.C.; Colwell, C.W. Polyethylene Contact Stresses, Articular Congruity, and Knee Alignment. Clin. Orthop. Relat. Res. 2001, 392, 232-238. [CrossRef] [PubMed]

37. Ishikawa, M.; Kuriyama, S.; Ito, H.; Furu, M.; Nakamura, S.; Matsuda, S. Kinematic alignment produces near-normal knee motion but increases contact stress after total knee arthroplasty: A case study on a single implant design. Knee 2015, 22, 206-212. [CrossRef]

38. Latypova, A.; Arami, A.; Becce, F.; Jolles-Haeberli, B.; Aminian, K.; Pioletti, D.P.; Terrier, A. A patient-specific model of total knee arthroplasty to estimate patellar strain: A case study. Clin. Biomech. 2016, 32, 212-219. [CrossRef] [PubMed]

39. Wilson, W.T.; Deakin, A.H.; Payne, A.P.; Picard, F.; Wearing, S.C. Comparative analysis of the structural properties of the collateral ligaments of the human knee. J. Orthop. Sports Phys. Ther. 2012, 42, 345-351. [CrossRef] 
40. Sugita, T.; Amis, A.A. Anatomic and biomechanical study of the lateral collateral and popliteofibular ligaments. Am. J. Sports Med. 2001, 29, 466-472. [CrossRef] [PubMed]

41. Trent, P.S.; Walker, P.S.; Wolf, B. Ligament Length Patterns, Strength, and Rotational Axes of the Knee Joint. Clin. Orthop. Relat. Res. 1976, 117, 263-270. [CrossRef]

42. Wijdicks, C.A.; Ewart, D.T.; Nuckley, D.J.; Johansen, S.; Engebretsen, L.; LaPrade, R.F. Structural properties of the primary medial knee ligaments. Am. J. Sports Med. 2010, 38, 1638-1646. [CrossRef] [PubMed]

43. Marinozzi, G.; Pappalardo, S.; Steindler, R. Human Knee Ligaments: Mechanical Tests and Ultrastructural Observations. Ital. J. Orthop. Traumatol. 1983, 9, 231-240.

44. Shu, L.; Yao, J.; Yamamoto, K.; Sato, T.; Sugita, N. In vivo kinematical validated knee model for preclinical testing of total knee replacement. Comput. Biol. Med. 2021, 132, 104311. [CrossRef] [PubMed]

45. Navacchia, A.; Rullkoetter, P.J.; Schütz, P.; List, R.B.; Fitzpatrick, C.K.; Shelburne, K.B. Subject-specific modeling of muscle force and knee contact in total knee arthroplasty. J. Orthop. Res. 2016, 34, 1576-1587. [CrossRef]

46. Freed, R.D.; Simon, J.C.; Knowlton, C.B.; Orozco Villaseñor, D.A.; Wimmer, M.A.; Lundberg, H.J. Are Instrumented Knee Forces Representative of a Larger Population of Cruciate-Retaining Total Knee Arthroplasties? J. Arthroplast. 2017, 32, $2268-2273$. [CrossRef]

47. Kutzner, I.; Bender, A.; Dymke, J.; Duda, G.; von Roth, P.; Bergmann, G. Mediolateral force distribution at the knee joint shifts across activities and is driven by tibiofemoral alignment. Bone Jt. J. 2017, 99-B, 779-787. [CrossRef]

48. Ledingham, J.; Regan, M.; Jones, A.; Doherty, M. Radiographic patterns and associations of osteoarthritis of the knee in patients referred to hospital. Ann. Rheum. Dis. 1993, 52, 520-526. [CrossRef]

49. Fiocchi, A.; Condello, V.; Madonna, V.; Bonomo, M.; Zorzi, C. Medial vs lateral unicompartmental knee arthroplasty: Clinical results. Acta Biomed. 2017, 88, 38-44.

50. Trepczynski, A.; Kutzner, I.; Kornaropoulos, E.; Taylor, W.R.; Duda, G.N.; Bergmann, G.; Heller, M.O. Patellofemoral joint contact forces during activities with high knee flexion. J. Orthop. Res. 2012, 30, 408-415. [CrossRef] [PubMed]

51. Dahlkvist, N.J.; Mayo, P.; Seedhom, B.B. Forces during Squatting and Rising from a deep squat. Eng. Med. 1982, 11,69-76. [CrossRef] [PubMed]

52. Reilly, D.T.; Martens, M. Experimental Analysis of the Quadriceps Muscle Force and Patello-Femoral Joint Reaction Force for Various Activities. Acta Orthop. Scand. 1972, 43, 126-137. [CrossRef]

53. Taylor, W.R.; Schütz, P.; Bergmann, G.; List, R.; Postolka, B.; Hitz, M.; Dymke, J.; Damm, P.; Duda, G.; Gerber, H.; et al. A comprehensive assessment of the musculoskeletal system: The CAMS-Knee data set. J. Biomech. 2017, 65, 32-39. [CrossRef]

54. Fox, A.J.S.; Wanivenhaus, F.; Rodeo, S.A. The basic science of the patella: Structure, composition, and function. J. Knee Surg. 2012, 25, 127-141. [CrossRef]

55. Lum, Z.C.; Saiz, A.M.; Pereira, G.C.; Meehan, J.P. Patella Baja in Total Knee Arthroplasty. J. Am. Acad. Orthop. Surg. 2020, 28, 316-323. [CrossRef] [PubMed] 